\title{
Skill Underutilization is Associated with Higher Prevalence of Hypertension: The Watari Study
}

\author{
Satoshi Konno and Masanori MunaKaTA \\ Preventive Medical Center and Division of Hypertension, Tohoku Rosai Hospital, Japan
}

\begin{abstract}
Skill Underutilization is Associated with Higher Prevalence of Hypertension: the Watari Study: Satoshi Konno, et al. Preventive Medical Center and Division of Hypertension, Tohoku Rosai Hospital-Objectives: The aim of this study was to investigate the association between skill underutilization, a psychological job stressor proposed in the $\mathrm{NIOSH}$ (National Institute for Occupational Safety and Health) job stress model, and the prevalence of hypertension. Methods: We studied sitting blood pressure and fasting blood laboratory parameters in 3,429 inhabitants of the town of Watari during annual health check-ups in 2010. A questionnaire was administered to examine job status, weekly working hours and degree of skill utilization. We divided subjects into tertiles according to skill utilization score. Multivariate logistic regression analysis was performed using prevalent hypertension as the dependent variable. Results: Questionnaires were collected from 2,550 individuals. After excluding those who were not in paid employment and those with missing data, 1,075 permanent or part-time workers were included in the final analysis. The odds ratio for the presence of hypertension was significantly higher in the lowest tertile of skill utilization score compared with the highest tertile $(1.776$; $95 \%$ confidence interval, 1.137-2.796) after adjusting for age, sex, body mass index, low-density lipoprotein cholesterol level, triglyceride level, current smoking status and daily alcohol intake. Conclusions: Skill underutilization was associated with a higher prevalence of hypertension in a working population including various kinds of occupations.
\end{abstract}

(J Occup Health 2014; 56: 225-228)

Key words: Hypertension, Occupational health, Psychological stress, Sex differences, Workload

A number of previous studies have reported an

Received Jul 1, 2013; Accepted Jan 16, 2014

Published online in J-STAGE Mar 4, 2014

Correspondence to: M. Munakata, Division of Hypertension, Tohoku

Rosai Hospital, 4-3-21 Dainohara, Aoba-ku, Sendai, Miyagi 981-

8563, Japan (e-mail: munakata@tohokuh.rofuku.go.jp) association between work-related stress and cardiovascular diseases $^{1-3)}$ or their risk factors such as hyperten$\operatorname{sion}^{4,5)}$. One of the most frequently used models to evaluate job stress in these studies has been Karasek's "job strain" model ${ }^{6}$, which defines high job strain as a combination of high psychological job demands and low job control. Another model for evaluating job stress is the National Institute for Occupational Safety and Health (NIOSH) job stress model ${ }^{7)}$, in which skill underutilization is proposed as a job stressor that can elicit acute stress reactions. Skill underutilization is defined as a condition in which a worker is unable to use his/her skills, leading to feelings of dissatisfaction. We have recently reported that perceived skill underutilization is associated with an increased risk for depressive trend ${ }^{8)}$. Moreover, in our preliminary study conducted with hospital employees (data not shown), we found that skill underutilization was an independent risk factor for cardiovascular events among several job stress components in the NIOSH questionnaire. To our knowledge, however, there has been no report on the relationship between skill underutilization and established cardiovascular risk factors. These observations have led us to examine skill utilization as a potential cardiovascular risk factor. The objective of this study was to investigate the association between the degree of skill utilization at work and the prevalence of hypertension.

\section{Subjects and Methods}

This study was conducted as a part of the Watari study, a community-based cohort study aimed at examining the association between qualitative and quantitative job stress and cardiovascular diseases. Detailed descriptions of the study have been provided previously ${ }^{8,9)}$. The study protocol was approved by the ethics committee of Tohoku Rosai Hospital, and all the participants provided written informed consent.

In this cross-sectional analysis, 3,020 people with Japanese National Health Insurance managed by the town of Watari and 409 local governmental employees 
were included. The former included self-employed workers (in areas such as agriculture, fisheries, forestry, retail trade, etc.), retired employees and their dependents; the latter included people working for the town office and its branch.

Demographic and anthropometric data were obtained, and fasting blood samples were collected after an overnight fast. Sitting blood pressure was measured once using an automated oscillometric sphygmomanometer (BX-10; Omron Colin, Kyoto, Japan) after a 5-min rest. Information on job status, weekly working hours (including overtime work) and lifestyle was collected using a questionnaire. Skill utilization score (ranging from 3 to 15) was calculated as the sum of the following 3 questions from the NIOSH job stress questionnaire: (1) How often does your job let you use the skills and knowledge you learned in school? (2) How often are you given a chance to do the things you do the best? (3) How often can you use skills from your previous experience and training? Answers to each question were scored from 1 to 5 (where 1=rarely, 2=occasionally, 3 =sometimes, 4=fairly often, and 5=very often).

\section{Statistical analysis}

All clinical data are expressed as means \pm standard deviation or as percentages. Subjects were divided into tertiles according to skill utilization score (lowest, 3 to 5; middle, 6 to 9; highest, 10 to 15 ), and differences across the groups were assessed using analysis of variance (ANOVA) or the chi-square test.
Multivariate logistic regression analysis was conducted using hypertension as the dependent variable. Hypertension was defined as a sitting blood pressure of $\geq 140 / 90 \mathrm{mmHg}$ or the use of antihypertensive medication. Odds ratios (OR) and $95 \%$ confidence intervals (CI) were calculated after adjusting for age, sex, body mass index (BMI, weight in $\mathrm{kg} / \mathrm{height}$ in meters squared), low-density lipoprotein (LDL) cholesterol levels, triglyceride levels, current smoking status and daily alcohol intake (g/day). The highest tertile of skill utilization score served as the reference group. The same analyses were conducted separately for men and women. Statistical analysis was performed using JMP (version 9.0 for Windows; SAS Institute, Cary, $\mathrm{NC}$, USA). A $p$-value of $<0.05$ was considered statistically significant.

\section{Results}

Out of 6,990 eligible individuals, 3,429 (49.1\%) participated in the check-up, and 2,550 answered the questionnaire. After excluding those with missing data $(n=249)$ and those who were not in paid employment $(n=1,226), 1,075$ permanent or part-time workers (751 self-employed workers and 324 local governmental workers) were included in the final analysis. The Cronbach's alpha for the skill utilization scale items was 0.847 .

Table 1 shows the clinical characteristics by tertiles of skill utilization score. The frequency of hypertension, subject age and LDL cholesterol levels were higher in those with lower skill utilization scores.

Table 1. Clinical characteristics by tertiles of skill utilization score

\begin{tabular}{lcccc}
\hline Variables & $\begin{array}{c}\text { Highest } \\
(\mathrm{n}=330)\end{array}$ & $\begin{array}{c}\text { Middle } \\
(\mathrm{n}=413)\end{array}$ & $\begin{array}{c}\text { Lowest } \\
(\mathrm{n}=332)\end{array}$ & $p$ value \\
\hline Skill utilization score & $12.6 \pm 1.8$ & $7.5 \pm 1.2$ & $3.8 \pm 0.7$ & \\
Age, years & $49.9 \pm 15.3$ & $51.2 \pm 14.1$ & $53.2 \pm 13.1$ & 0.010 \\
Men, \% & 46.1 & 53.0 & 39.5 & 0.001 \\
BMI, kg/m & $23.0 \pm 3.3$ & $23.1 \pm 3.1$ & $23.4 \pm 3.4$ & 0.275 \\
Systolic BP, mmHg & $119.4 \pm 16.1$ & $120.9 \pm 15.7$ & $122.2 \pm 16.3$ & 0.087 \\
Diastolic BP, mmHg & $70.4 \pm 11.7$ & $71.6 \pm 11.7$ & $71.9 \pm 11.2$ & 0.210 \\
LDL cholesterol, mg/d $l$ & $111.2 \pm 30.0$ & $115.6 \pm 29.6$ & $117.2 \pm 29.8$ & 0.026 \\
HDL cholesterol, mg/d $l$ & $63.7 \pm 15.7$ & $62.3 \pm 15.7$ & $64.0 \pm 15.8$ & 0.271 \\
Triglycerides, mg/d $l$ & $95.4 \pm 56.4$ & $105.2 \pm 69.5$ & $100.0 \pm 54.2$ & 0.095 \\
HbA1c, \% & $5.8 \pm 0.7$ & $5.8 \pm 0.7$ & $5.9 \pm 0.8$ & 0.178 \\
Current smoking, \% & 21.5 & 23.7 & 19.3 & 0.340 \\
Alcohol intake, g/day & $10.6 \pm 19.9$ & $9.4 \pm 18.3$ & $8.4 \pm 18.0$ & 0.326 \\
Weekly working hours, h & $38.0 \pm 16.2$ & $38.2 \pm 14.8$ & $37.1 \pm 15.7$ & 0.615 \\
Hypertension, \% & 19.1 & 21.8 & 28.0 & 0.019 \\
\hline
\end{tabular}

Data are presented as means \pm standard deviation or percentages. $p$ value, analysis of variance or the chi-square test. BMI, body mass index; BP, blood pressure; LDL, low-density lipoprotein; HDL, high-density lipoprotein; HbA1c, glycated hemoglobin. 
Table 2. Baseline characteristics for men and women stratified by tertiles of skill utilization score

\begin{tabular}{|c|c|c|c|c|c|c|c|c|}
\hline \multirow[b]{2}{*}{ Variables } & \multicolumn{4}{|c|}{ Men $(n=502)$} & \multicolumn{4}{|c|}{ Women $(n=573)$} \\
\hline & $\begin{array}{l}\text { Highest } \\
(\mathrm{n}=152)\end{array}$ & $\begin{array}{l}\text { Middle } \\
(\mathrm{n}=219)\end{array}$ & $\begin{array}{l}\text { Lowest } \\
(n=131)\end{array}$ & $p$ value & $\begin{array}{l}\text { Highest } \\
(\mathrm{n}=178)\end{array}$ & $\begin{array}{c}\text { Middle } \\
(\mathrm{n}=194)\end{array}$ & $\begin{array}{l}\text { Lowest } \\
(\mathrm{n}=201)\end{array}$ & $p$ value \\
\hline Age, years & $54.3 \pm 14.6$ & $53.5 \pm 14.4$ & $54.9 \pm 13.7$ & 0.647 & $46.1 \pm 14.9$ & $48.7 \pm 13.4$ & $52.2 \pm 12.6$ & $<0.001$ \\
\hline BMI, $\mathrm{kg} / \mathrm{m}^{2}$ & $23.9 \pm 2.9$ & $23.8 \pm 3.1$ & $23.9 \pm 3.1$ & 0.913 & $22.2 \pm 3.4$ & $22.3 \pm 3.0$ & $23.0 \pm 3.6$ & 0.025 \\
\hline Systolic BP, mmHg & $123.7 \pm 15.4$ & $123.8 \pm 15.3$ & $125.3 \pm 16.1$ & 0.602 & $115.8 \pm 15.9$ & $117.6 \pm 15.5$ & $120.2 \pm 16.2$ & 0.027 \\
\hline Diastolic BP, mmHg & $74.6 \pm 11.4$ & $74.1 \pm 11.3$ & $74.7 \pm 11.0$ & 0.888 & $66.9 \pm 10.8$ & $68.7 \pm 11.4$ & $70.1 \pm 11.1$ & 0.019 \\
\hline LDL cholesterol, $\mathrm{mg} / \mathrm{d} l$ & $111.6 \pm 30.3$ & $116.0 \pm 27.6$ & $113.7 \pm 27.3$ & 0.338 & $110.8 \pm 29.8$ & $115.2 \pm 31.7$ & $119.4 \pm 31.2$ & 0.027 \\
\hline HDL cholesterol, $\mathrm{mg} / \mathrm{d} l$ & $57.2 \pm 14.0$ & $56.4 \pm 15.1$ & $57.7 \pm 12.2$ & 0.685 & $69.3 \pm 15.0$ & $68.9 \pm 13.7$ & $68.1 \pm 16.6$ & 0.734 \\
\hline Triglycerides, mg/d $l$ & $116.9 \pm 64.0$ & $127.6 \pm 79.8$ & $118.0 \pm 60.8$ & 0.278 & $77.1 \pm 41.2$ & $79.9 \pm 43.8$ & $88.3 \pm 46.0$ & 0.033 \\
\hline $\mathrm{HbA} 1 \mathrm{c}, \%$ & $5.9 \pm 0.6$ & $5.9 \pm 0.9$ & $5.9 \pm 0.9$ & 0.948 & $5.7 \pm 0.7$ & $5.7 \pm 0.6$ & $5.8 \pm 0.8$ & 0.026 \\
\hline Current smoking, $\%$ & 37.5 & 35.6 & 36.6 & 0.932 & 7.9 & 10.3 & 8.0 & 0.630 \\
\hline Alcohol intake, g/day & $20.1 \pm 24.2$ & $15.8 \pm 21.8$ & $19.1 \pm 23.8$ & 0.168 & $2.5 \pm 9.7$ & $2.2 \pm 9.0$ & $1.5 \pm 6.6$ & 0.510 \\
\hline Weekly working hours, $\mathrm{h}$ & $42.2 \pm 17.0$ & $39.5 \pm 15.8$ & $40.8 \pm 15.0$ & 0.271 & $34.5 \pm 14.5$ & $36.7 \pm 13.5$ & $34.7 \pm 15.7$ & 0.270 \\
\hline Hypertension, $\%$ & 27.6 & 29.7 & 36.6 & 0.230 & 11.8 & 12.9 & 22.4 & 0.007 \\
\hline
\end{tabular}

Data are presented as means \pm standard deviation or percentages. $p$ value, analysis of variance or the chi-square test. BMI, body mass index; BP, blood pressure; LDL, low-density lipoprotein; HDL, high-density lipoprotein; HbA1c, glycated hemoglobin.

However, when we analyzed data for men and women separately (Table 2), we found a positive association between age and lower skill utilization scores in women, but not in men. BMI, systolic and diastolic blood pressure, LDL cholesterol levels, triglyceride levels, glycated hemoglobin levels and the prevalence of hypertension were also higher in the lower tertiles of skill utilization scores in women. On the other hand, differences in clinical characteristics across tertile groups were not significant in men.

Multivariate logistic regression analyses adjusted for age, sex, BMI, LDL cholesterol levels, triglyceride levels, current smoking status and daily alcohol intake demonstrated that the OR for the presence of hypertension was significantly higher in the lowest tertile compared with the highest tertile $(1.776 ; 95 \% \mathrm{CI}$ : 1.137-2.796). When similar analyses were conducted separately in men and women, the result remained significant in men (OR 1.847; 95\% CI: 1.007-3.419) but not in women (OR 1.683; 95\% CI: 0.859-3.384).

\section{Discussion}

The results of this study indicated for the first time that skill underutilization, a job stressor that has drawn less attention than other psychological job stress factors, is independently associated with a higher prevalence of hypertension in a working population including various kinds of occupations.

We observed a positive association between age and lower skill utilization scores in only women. On the other hand, after adjustment for possible confounders, the association between skill underutilization and prevalent hypertension was significant in men but not in women. A number of studies have demonstrated a positive relationship between high job strain and increased risk for hypertension in $\operatorname{men}^{5,10,11)}$, while the relationship has not been fully elucidated in women. Our data are in line with those in previous reports. Although the reason for this gender difference is unclear, the following mechanisms may be involved. First, there may be gender differences in occupational characteristics and status, which are known to influence the prevalence of hypertension ${ }^{12}$. Second, the prevalence of hypertension in men $(30.9 \%)$ was approximately double that in women $(15.9 \%)$ in our study population, which may have limited the statistical power of the analyses. Another possible explanation is a gender difference in pressor response to psychological stress. We have previously reported in untreated hypertensive patients that men showed a significantly greater blood pressure increase in response to experimental psychological stress than did women ${ }^{13)}$.

Our study has several limitations. First, the present analyses were not based on the job strain model or the effort-reward imbalance model ${ }^{14)}$, the two most frequently used models for evaluating job-related stress; thus, a direct comparison between our findings and those of previous studies was not possible. In addition, we have no information on other psychological factors, such as job control or social support, which may partly confound the relationship between skill utilization and the presence of hypertension. Second, types of jobs, employment status (permanent or parttime) and occupational social class were not considered in the present study. Moreover, we did not 
collect information on stress at home, which has been reported to have a greater impact on health in women than in men ${ }^{15}$. Any of these factors could have influenced the results of this study. Third, blood pressure was assessed through casual measurement, which is less reliable for the evaluation of work site blood pressure than ambulatory blood pressure measurement. Finally, we have to consider that participants in municipal annual health check-ups do not necessarily represent the general working population, and further investigations should be conducted to determine the generalizability of our findings.

In conclusion, a low perceived degree of skill utilization was associated with a higher prevalence of hypertension in a working population including various kinds of occupations. Our results may provide new information relevant to the establishment of healthier workplaces. Further longitudinal studies with a larger population are needed to confirm the causal relationship between skill underutilization and incident hypertension.

Acknowledgment: This study was supported by Grants-in-Aid from the Japan Labor, Health, and Welfare Organization.

\section{References}

1) Schnall PL, Landsbergis PA, Baker D. Job strain and cardiovascular disease. Annu Rev Public Health 1994; 15: 381-411.

2) Bosma $H$, Marmot MG, Hemingway $H$, et al. Low job control and risk of coronary heart disease in Whitehall II (prospective cohort) study. BMJ 1997; 314: 558-65.

3) Rosengren A, Hawken S, Ounpuu S, et al. Association of psychosocial risk factors with risk of acute myocardial infarction in 11119 cases and 13648 controls from 52 countries (the INTERHEART study): case-control study. Lancet 2004; 364: 953-62.

4) Schnall PL, Pieper C, Schwartz JE, et al. The relationship between 'job strain,' workplace diastolic blood pressure, and left ventricular mass index. Results of a case-control study. JAMA 1990; 263: 1929-35.

5) Radi S, Lang T, Lauwers-Cances V, et al. Job constraints and arterial hypertension: different effects in men and women: The IHPAF II case control study. Occup Environ Med 2005; 62: 711-7.

6) Karasek RA. Job demands, job decision latitude and mental strain: Implications for job redesign. Admin Sci Q 1979; 24: 285-307.

7) Hurrell JJ, Jr., McLaney MA. Exposure to job stress-a new psychometric instrument. Scand J Work Environ Health 1988; 14 (Suppl 1): 27-8.

8) Konno S, Hattori $T$, Sato $T$, et al. Relationship between job stress and depressive trend in the general population: the Watari study. Jpn J Occup Med Traumatol 2013; 61: 133-7.

9) Munakata M, Konno S, Ohshima M, Ikeda T, Miura $\mathrm{Y}$, Ito S. High-normal blood pressure is associated with microalbuminuria in the general population: the Watari study. Hypertens Res 2011; 34: 1135-40.

10) Light KC, Turner JR, Hinderliter AL. Job strain and ambulatory work blood pressure in healthy young men and women. Hypertension 1992; 20: 214-8.

11) Cesana G, Sega R, Ferrario M, et al. Job strain and blood pressure in employed men and women: a pooled analysis of four northern Italian population samples. Psychosom Med 2003; 65: 558-63.

12) Wiernik E, Pannier B, Czernichow $S$, et al. Occupational status moderates the association between current perceived stress and high blood pressure: Evidence from the IPC cohort study. Hypertension 2013; 61: 571-7.

13) Munakata M, Saito $Y$, Nunokawa $T$, et al. Clinical significance of blood pressure response triggered by a doctor's visit in patients with essential hypertension. Hypertens Res 2002; 25: 343-9.

14) Siegrist J. Adverse health effects of high-effort/ low-reward conditions. J Occup Health Psychol 1996; 1: 27-41.

15) Orth-Gomer K, Wamala SP, Horsten M, et al. Marital stress worsens prognosis in women with coronary heart disease: The Stockholm Female Coronary Risk Study. JAMA 2000; 284: 3008-14. 